\title{
ENCEFALOPATIA HIPERTENSIVA SEUDOTUMORAL
}

\author{
Ignacio de Gispert Cruz *
}

Llama la atención al hecho de que a pesar de su importancia y relativa frecuencia, la encefalopatia por hipertensión maligna apenas se cita en las obras de neurologia y hasta de medicina. Todavia es más acentuado este silencio para la forma seudotumoral a pesar de que la confusión diagnóstica de la encefalopatia con un tumor cerebral u otro proceso expansivo intracraneal es en la practica muy posible.

El término de encefalopatia hipertensiva fué empleado por primera vez por Oppenheimer y Frishberg quienes comprendian bajo esta denominación aquellos casos de enfermedad hipertensiva con manifestaciones predominantes cerebrales independientemente de la presencia o ausencia de lesiones renales. Los escasos autores que han llamado la atención en estos últimos años sobre esta afección emplean las denominaciones más diversas. Asi, Scheinker la llama "enfermedad hipertensiva del cerebro", Thiebault, "forma cerebral de la hipertensión arterial"; Goodman propone la substitución del nombre de encefalopatia hipertensiva por el de "trom. bosis cerebral recurrente hipertensiva" y recientemente Russek y Zohman la denominan "encefalopatia vascular". Todos ellos coinciden en la exis. tencia de lesiones vasculares debidas fundamentalmente a la hipertensión endocraneal provocada a su vez por la vasoconstricción y edema cerebral consiguiente.

Es también limitado el número de trabajos sobre el diagnóstico diferencial entre tumor cerebral y encefalopatia hipertensiva (Ketterer, Griffith). Ultimamente Holub, Starling y colab., Chavany, Lopez Fernandez y otros han señalado las dificultades existentes en algunos casos para diferenciar ambos procesos. Dereux los califica de "hipertensión arterial seudotumoral". Finalmente estas dificultades han sido reconocidas por algunos neurocirujanos (Vincent, Cushing, Puech) constituyendo este tema una de las ponencias del Congreso Sudamericano de Neurocirujia de 1945.

La encefalopatia hipertensiva puede constituir una complicación de la hipertensión simple o enfermedad hipertensiva esencial o bien ser debida a otros procesos renales, endocrinos y cardio-vasculares. Algunos de estos casos pueden desarrollar el cuadro clinico de un tumor cerebral en que

* Jefe del Departainento de Neurologia del Hospital de San Juan de Dios y del Servicio de Neurologia de la Clínica Médica Universitária B. (Barceloní España). 
las cefalalgias, vómitos, vértigos $\mathrm{y}$, sobre todo, las alteraciones de fondo de ojo pueden llevar a un error diagnóstico. Aunque corrientemente faltan signos precisos de localización es posible que las lesiones cerebro-vasculares debidas a la hipertensión predominen en determinadas zonas del encéfalo. Es entonces cuando estos signos focales a menudo de caracter progresivo (hemiplejia, afasia, hemianopsia, paralisis diversas, etc.) pueden hacer pensar en un proceso expansivo intracraneal (tumor, aracnoiditis, abceso, hematoma, etc.), discutiendose la conveniencia de determinados examenes complementarios y, en último término, la intervención.

En este último grupo pueden incluirse dos tipos de seudotumores: los debidos a hipertensión esencial y los debidos a la hipertensión por otras causas, sobre todo por lesiones renales. Entre estas se considerarian principalmente las afecciones de tipo de la glomerulonefritis crónica y la esclerosis renal intercapilar de Kimmenstiel y Wilson. En la practica es frecuente que el proceso renal puede pasar desapercebido predominando las manifestaciones cerebrales. Es posible también, en la práctica neurologica, que en enfermos diagnosticados a veces demasiado rapidamente de tumor intracraneal o simplemente de proceso expansivo intracraneal, el simple examen de la presión sanguinea o la existencia de albumina en la orina nos pueda orientar por el buen camino.

\section{HIPERTENSION ESENCIAL}

Las complicaciones de la hipertonia esencial son diversas pero puede admitirse fundamentalmente que todas tienen un origen vascular: el espasmo seguido de arteriolitis con isquemia cerebral y edema subsiguientes. Las muertes por hipertensión arterial se dividirian, segun las recientes estadísticas de Griep y colaboradores, aproximadamente por igual entre complicaciones cerebrovasculares y cardiacas. En cambio menos veces fallecen estos enfermos por insuficiencia renal y uremia ulterior, pero, con frecuencia pueden desarrollar la encefalopatia hipertensiva con todas sus consecuencias. La posibilidad de marcadas lesiones esclerosas arteriolares cerebrales con integridade total o con lesiones minimas de los vasos renales al cabo de muchos años de la enfermedad hipertensiva, ha sido puesta de manifiesto por Davidson y Brill, Bordley y otros.

Las complicaciones renales suelen solo presentarse en periodos avanzados de la enfermedad, constituyendo entonces las pruevas de descarga de urea un indice pronostico de un valor extraordinario. Cuando la descarga de urea es inferior al 50 por ciento de lo normal la cifra de mortalidad seria elevada y precoz. En cuanto a la albuminuria, solo se observaria cuando ya se han producido complicaciones cardiovasculares de cierta importancia.

En cuanto a los problemas etiopatogenicos de la que se ha convenido en llamar hipertensión esencial, término con el que siempre encubrimos 
nuestra ignorancia, es evidente que son muchos y complicados. Además del factor renal y cardiovascular citados existen otros como las alteraciones de las glándulas suprarrenales que desenpeñan en algunos casos un papel evidente. El ejemplo que más recientemente ha llamado la atención es el tumor hiperplásico, hipernefroma o paraganglioma segun se origine de la substancia cortical o medular de la glandula suprarrenal. En estos casos la encefalopatia suele ir asociada a otras manifestaciones patologicas (hirsutismo, sindrome adiposo-genital, pubertad precoz, etc.) que si pueden constituir un sindrome similar al de Cushing, pueden, por otra parte, ser muy discretos.

En la enfermedad de Cushing propiamente dicha por adenoma basofilo del lobulo anterior de la hipofisis, es posible también un sindrome seudotumoral cerebral debido a la hipertensión arterial. En una observación nuestra ya publicada, las cefalalgias dominavan el cuadro. Es posible, por otra parte, que los signos iniciales sean los de la hipertensión faltando o siendo muy discretos los otros sintomas de la enfermedad (estrias abdominales, osteoporosis, atrofia genital, hirsutismo, alteraciones del metabolismo del calcio y colesterina, etc.).

En los casos en que faltan alteraciones renales algunos autores han llegado a admitir que, en la encefalopatia, el cerebro isquémico seria capaz de elaborar una substancia hipertensora similar a la que se produce en la insuficiencia renal. Además de la hipertensión, el hecho constante en la encefalopatia es el espasmo vascular, la esclerosis arteriolar (principalmente cerebral y renal), la isquemia cerebral y el edema. Para Pickering la base de los trastornos agudos seria la obstrucción arterial de una manera similar a lo que sucede en la embolia cerebral de origen cardiaco. En ambos casos el restablecimiento de la circulación a través de vasos colaterales explicaria la posible transitoriedad de los trastornos.

Para el mecanismo de la encefalopatia pueden invocarse los distintos factores admitidos para la hipertensión en general: a - aumento de resistencia periférica (angioespasmo periférico por factores neurohormonales, suprarrenal y hipofisario); b - aumento de la cantidad de sangre como sucederia en el caso del individuo plétorico; $\mathrm{c}$ - alteraciones de los diversos órganos y centros vegetativos que regulan o tienen influencia sobre la presion arterial (bulbo, diencefalo, corteza). De estos últimos destacarian la importancia de las lesiones de la substancia reticular del bulbo que provocan descensos rapidamente mortales de la presión arterial.

Han de tenerse también en cuenta las frecuentes variaciones en las cifras de presion que pueden aparecer normales o casi normales en el momento de ser reconocido el paciente. Finalmente la posibilidad de hipertensiones localizadas, hecho muy discutido pero que tiende a ser admitido por diversos autores. Por otra parte, como señala Cañizo, "no pueden establecerse barreras infranqueables o limites exactos entre hipertonia esencial, lesiones vasculares difusas y nefroesclerosis benignas y malignas. Solo existen diferencias de grado y de unos se puede pasar a otros, unas veces 
de un modo lento y otras de un modo extraordinariamente rapido y maligno". En resumen pues, el mecanismo de la hipertensión y encefalopatia es sumamente complejo en el que pueden intervenir una serie de factores vasculares, locales, tóxicos, psiquicos, constitucionales, hormonales y que tienen en algunos casos una influencia indiscutible.

\section{SINTOMATOLOGIA}

La encefalopatia puede manifestarse por la aparición brusca de uno o más sintomas (crisis apopletica, cefalalgias, hemiplejia, afasia, pérdida de visión, etc.) o, lo que es más corriente, hacerlo de una manera sumamente lenta. En este último caso y generalmente en personas jovenes o de mediana edad, aparecen vértigos, somnolencia, parestesias, estado nauseoso, moscas volantes, fatigabilidad y malestar general. A menudo también refiere el paciente una disminución notable de su capacidad psiquica. Todos estos trastornos pueden desaparecer temporalmente para reaparecer de nuevo. La variabilidad de sintomas es uno de los caracteres más constantes de la afección en sus comienzos.

Las cefalalgias constituyen uno de los sintomas más constantes y es por este motivo que el paciente recurre generalmente al especialista. Puede presentar las localizaciones más diversas (frontal, occipital o en la región de la nuca). El dolor puede ser unilateral confundiendose a menudo con la jaqueca. Aunque el dolor suele ser continuo, no es raro que presente algunas remisiones en intensidad. En las formas agudas (ataque cerebral hipertensivo de Mc Alpine) aparecen de una manera brusca una serie de sintomas desde cefalalgia a coma y convulsiones. No existe retención nitrogenada $\mathrm{y}$, segun la sintomatologia, las divide Mc Alpine en formas con signos de edema cerebral y formas sin estos signos. En el primer tipo frecuente en hombres de menos de 40 años se observan cefalalgias, convulsiones, retinopatia y aumento brusco de la presión arterial. Las formas sin signos de edema cerebral, aparecen generalmente en individuos de 40 a 60 años. La hipertensión es permanente y existieron casi siempre signos anteriores en forma de parestesias, afasias, paresias, etc. Antes del coma el paciente suele estar omnubilado o inconsciente. A menudo el coma va precedido de algunas convulsiones con signo de Babinskj bilateral. A veces el ataque solo va precdido de un periodo de cefalalgias o de crisis jacksonianas a veces muy limitadas. Falta estasis papilar y, aunque la cifra de urea de la sangre y nitrógeno no proteico sean nor. males, puede hallarse un grado moderado de albuminuria. Algunos de estos casos convulsivos de encefalopatia han sido considerados durante has. tante tiempo como epilepsias esenciales o seniles.

Los trastornos de psiquismo, en mayor o menor grado, pueden considerarse como constantes. En general consistem en una disminución global de la capacidad intelectual. Los familiares o el mismo enfermo re- 
fieren sus cambios de caracter, inatención, pérdida de memoria. Algunoc de estos enfermos muestran un grado marcado de infantilismo. A medida que progresan las lesiones cerebrales ocluyendose nuevas crterias y arteriolas se acentúa el deficit mental debiendo pensarse en estos casos en una agravación no solamento de la encefalopatia sino de la enfermedad hipertensiva en general. Algunos de estos pacientes jovenes han sido considerados como esquizofrénicos durante bastante tiempo antes de descubrir la encefalopatia. En enfermos de edad madura se piensa a menudo en la posibilidad de una paralisis general; en los viejos, en la demencia senil o en la encefalopatia arterioesclerósica.

A veces las crisis angioespasticas que originaran una hemiplejia o afasia pueden ir precedidas de un periodo de estado confusional psíquico. En una observación de Alsina Bofill de un enfermo de 52 años sobreviene bruscamente un estado de subdelirio que recuerda una crisis maniaca; solo aparece la hemiplejia y afasia al cabo de 15 dias.

Entre los sintomas motores irritativos se encuentran las crisis corvulsivas que pueden ser generalizadas o, lo que es más frecuente, hemilaterales o comprender una sola extremidad (Russek y Zohman). En los casos en que se produce pérdida de conocimiento puede persistir esta desde algunos minutos a varias horas. Para Sterling las convulsiones serian bastante menos frecuentes en la encefalopatia hipertensiva que en los tumores intracraneales. Entre los trastornos deficitarios motores nos encontramos con paresias o paralisis unilaterales corrientemente de caracter transitorio aunque más adelante, con la producción de nuevos brotes angicespasticos, pueden hacerse definitivas.

Las alteraciones de fondo de ojo son casi constantes en la encefalopatia y suelen presentarse en mayor o menor grado en uno u otro periodo de la hipertensión maligna. Son tan frecuentes sin embargo las divergencias que hallamos en los dictámenes de fondo de ojo de estos enfermos que hemos llegado a la conclusión que pocas veces nos hallamos ante una sintomatologia precisa. Se califican las alteraciones halladas de papilitis, retinopatia, estasis papilar, neurorretinitis, hemorragias peripapilares, etc., sin que se observe una retinopatia tipica hipertensiva. Segun nuestra experiencia hemos de confesar la frecuencia de formas de transición que dificilmente nos atrevemos a calificar de una manera absoluta. Sobre esta cuestión han insistido recientemente Sterling y colaboradores quienes han descrito 30 casos de hipertensión maligna con alteraciones de fondo de ojo. Reconocen las dificultades en algunos de ellos para establecer el diag. nostico diferencial con los tumores cerebrales ya que los caracteres del edema papilar pueden ser iguales en ambos procesos. Incluso puede darse el caso de estasis papilar de un lado con atrofia óptica del otro (sindrome de Foster-Kennedy) tal como sucede en una de nuestras observaciones.

Quizás podriamos admitir que el signo más importante diferencial entre tumor y encefalopatia hipertensiva seria no solo la evolución de los 
trastornos de fondo de ojo sino de los sintomas en general: en la encefalopatia, aparición brusca y remisión o muerte; en el tumor, evolución lenta $y$ progresiva. Esta regla of receria, sin embargo, numerosas excepciones.

La misma retinitis albuminúrica con sus caracteristicas manchas blancas situadas principalmente en la mácula también pueden confundirse en los casos atipicos con el simple edema de la papila: las manchas blancas pueden confluir y hasta formar un anillo peripapilar; en la mácula las manchas suelen ser radiadas en estrella. Para Weil la retinitis albuminúrica seria propia de la hiperazotemia mientras que el edema papilar se observaria más bien en la retención cloruremica y la hemorragia retiniana y peripapilar en la hipertensión esencial.

Aunque el grado de alteraciones de fondo de ojo constituye un indice pronóstico importante en los casos de encefalopatia y en general de la enfermedad hipertensiva, ha de tenerse en cuenta que la retinopatia o la estasis papilar no indican precisamente que haya de ocurrir un accidente cerebral ya que la muerte puede producirse por otras complicaciones cardiacas o renales. Sin embargo constituye un exponente del grado de degeneración vascular que acompaña a la enfermedad hipertensiva.

En lo que se refiere al liquido cefalorraquideo es frecuente en la hipertensión maligna un aumento de presión aunque no alcanze, por lo general, la cifra de los procesos expansivos intracraneales. En los casos comprobados por nosotros la composición del liquido fué siempre normal y las presiones aumentadas solo ligeramente. Sterling, en cambio, señala en los casos de encefalopatia con edema papilar, frecuentes modificaciones de la curva coloidal pero también con una composición normal.

No hemos de extendernos en consideraciones diagnosticas diferenciales con otros procesos neurologicos, aunque en algunos casos la encefalopatia hipertensiva ha podido confundirse con una encefalitis letargica, cor- Ia enfermedad de Schilder, con la esclerosis multiple. Ya hemos indicado también el parecido de algunas formas psiquicas de la encefalopatia con un sindrome esquizof rénico, demencial senil o con la paralisis general.

Para la distinción etiologica entre hipertonia esencial o secundaria a lesiones renales o a otros procesos es indispensable la estrecha colaboración con el medico internista. Lo fundamental es no desconocer la existencia de un proceso grave hipertensivo endocraneal de origen vascular que requiere a veces un tratamiento urgente e incluso una intervención quirurgica decompresiva cuando el cuadro clinico afecta gravemente la visión o amenaza la vida del enfermo.

A continuación resumimos algunas historias clinicas de enfermos afectos de enctfalopatia hipertensiva en que hubo de plantearse el diagnostico diferencial con un posible proceso expansivo intracraneal.

Caso 1 - Jaime B., de 50 años, profesión contable, sin antecedentes patológicos familiares importantes. Explica dos traumatismos craneales por caída, uno hace 30 años, el otro por caída de caballo hace unos 10 años sufriendo conmoción 
cerebral. Hace 4 años, hemorragia retiniana con perdida casi completa de la visión en ojo izquierdo, de la que ha ido recuperándose muy lentamente. Desde hace mucho tiempo hipaacusia del oído derccho. Desde hace dos meses y medio, persistente dolor de cabeza que se extiende desde la nuca a los ojos. Nota que pierde agudeza visual en el ojo derecho, con el que veia perfectamente. Ha tenido 4 o 5 ataques en los que queda rigido y pierde el conocimiento. Desde hace algunas semanas nota malos olores, especialmente de los alimentos, en los que encuentra siempre malgusto.

Exploración clinico-neurológica - Ligera propulsión globo ocular derecho; anosmia en el lado izquierdo e hiposmia a la derecha. No se descubren alteraciones de otros pares craneales. Reflejos, sensibilidad y motilidad normales. No existen signos cerebelosos. Fondo de ojo: estasis papilar derecho con focos hemorrágicos peripapilares y atrofia óptica en el ojo izquierdo (síndrome de FosterKennedy); reducción campos visuales (Prof. Soria). Hemograma normal. Urea en la sangre $0,40 \mathrm{~g}$; glucemia $1 \mathrm{~g}$; velocidad de sedimentación 72 a la primera hora; presión arterial 18-9. En la orina, indicios de albúmina, 10 hematies por cc y 4 leucócitos, cilindros hialianos y granulosos y algunas células renales. Pruebas de eliminacion renal normales.

Ante la posibilidad de la existencia de un tumor de fosa anterior se practican radiografias de craneo asi como la arteriografia y neumografia cerebrales, que dan resultados normales. El paciente es ingresado en el Hospital Clínico (Servicio Prof. Soriano), donde presenta varias crisis que coinciden con brotes hipertensivos que remiten a los 15 minutos del ataque. Ha presentado también varios accesos de dispnea inspiratoria que obligan a un tratamiento de urgencia; a la exploración, numerosos estertores de estasis en ambos campos pulmonares.

A pesar de habersele practicado la gangliectomia y esplancnicectomia el enfermo no experimenta mejoria alguna persistente y si solamente una ligera disminución de la presión arterial despues de los primeros dias de la intervención.

El interés del caso estriba principalmente en que la hipertensión, que podemos calificar de maligna esencial, originó un cuadro de encefalopatia. Además de un síndrome de hipertensión endocraneal con cefalalgias, crisis convulsivas, nos encontramos con un síndrome de Foster-Kennedy (estasis papilar de un lado con atrofia óptica del otro), asociado a la anosmia y protrusión del globo ocular apoyando la existencia de un tumor de fosa anterior, probablemente de fosa supraorbitaria o prefrontal. 'Todo ello ante una presión arterial al principio no muy exagerada, en ausencia de lesiones cardiovasculares y lesiones importantes renales permitia suponer la posibilidad de un proceso expansivo de fosa anterior craneal. Solo despues de la ventriculografia y arteriografia se pudo admitir de una manera definitiva que toda la sintomatologia se debia a la enfermedad hipertensiva.

La observación que sigue nos fué remitida por el Dr. Arruga con el diagnóstico de estasis papilar doble.

Caso 2 - Roberto $R$., de 49 años, sin antecedentes patológicos familiares importantes. Hace dos años acudió por primera vez al médico a causa de intensas cefalalgias, siendole advertido que "tenia presión". Desde entonces frecuentes e intensas cefalalgias, fatiga y malestar general. Ultimamente, pérdidas momentaneas de visión. Algunos vómitos de aparición brusca sin nauseas previas. Zumbidos de oídos. Desde hace algunas semanas experimenta dificultad para la marcha yendose indistintamente hacia uno $\mathbf{y}$ otro lado, pero con un ligero predominio para el laco izquierdo. Ha perdido memoria y capacidad intelectual y durante el interrogatório manifiesta un evidente retardo de psiquismo. Parestesias en la hemicara y lado izquierdos. Se le han hinchado algunas veces los tobillos.

Exploración clínico-Reurológica - Estnsis papilar doble muy marcado con algunas hemorragias peripapilares. Hipoacusia en el oído izquierdo. Resto de los 
pares craneales normales. Marcha irregular mas bien de tipo atáxico-cerebeloso pero con un ligero predominio para la desviación hacia la izquierda. Las pruebas cerebelosas (adiadocinesia, dismetria, etc.) resultan dificiles de explorar dada la bradipsiquia del paciente que titubea al hacerlas $\mathbf{y}$ presta poca atención a nuestras órdenes. Sin embargo, en las pruebas dedo-nariz y dedo-índice, la dismetria es evidente. Reflejos tendinosos, cutáneos y osteoperiósticos normales. Presión arterial 24-11. En la orina, albúmina intensamente positiva. Se indica la conveniencia de practicar exámenes complementarios de urea en la sangre y pruebas de eliminación renal. El enfermo desaparece $\mathrm{y}$ hemos sabido que falleció al cabo de unos tres meses despues de una crisis apopletiforme.

Aunque se trata de un caso examinado y estudiado incompletamente, ya que fue visto una sola vez en nuestra consulta, el cuadro de estasis papilar asociado a otros sintomas de hipertensión endocraneal hizo creer de momento que podia tratarse de un tumor intracraneal. La existencia de temblor, ataxia, dismetria, podian hacer pensar en una localización en fosa posterior. Quizás un tumor del ángulo, desde que a los signos cerebelosos se añadian los zumbidos de oído y disminución de la agudeza auditiva del lado derecho. Sin embargo, la presión arterial elevada, asi como la presencia de abundante cantidad de albúmina en la orina, apoyan fuertemente la idea de que nos encontramos ante una encefalopatia hipertensiva en que era dificil dilucidar si las probables lesiones renales eran primitivas o secundarias.

En la observación siguiente el cuadro de hipertensión endocraneal, así como las alteraciones de fondo de ojo con perdida progresiva y casi completa de la visión, justificaron la intervención quirúrgica. Sin embargo, fué escasa la acción de la intervención.

Caso 3 - Antonia S., de 32 años, casada, sin antecedentes familiares importantes. Neumonia hace 14 años. La enfermedad actual empezó hace once meses siendo el sintoma inicial una intensa cefalalgia de localización occipital y de tipo paroxístico, que cedia parcialmente con los analgésicos corrientes. Al cabo de cuatro meses aparecen frecuentes vómitos de tipo cerebral y vértigos de predominio matutino. Al mismo tiempo empieza a experimentar dismunición de la agudeza visual en ambos ojos. Durante estos últimos meses los trastornos visuales han experimentado regresiones transitorias. Sin embargo, en la actualidad refiere la enferma que está ciega del ojo derecho y que conserva la visión inalterada en el otro ojo.

Examen clínico-neurológico - Como trastornos subjetivos pueden citarse los vómitos de tipo cerebral que todavia persisten, vértigos, acúfenos en el oído izquierdo, intensas cefalalgias de localización principalmente occipital y amaurosis del ojo izquierdo. La exploración neurológica nos da motilidad, sensibilidad, reflejos y aparato cerebelosos normales. Examen de fondo de ojo - En O.D., extenso $\mathrm{y}$ intenso edema de la papila con profusión de hemorragias radiadas en la parte superior; papiledema y edema peripapilar que se pronuncia por el sector temporal; hasta la periferia la retina no presenta caracteres normales y la circulación retiniana pierde color y calibre. En O.I., pronunciado edema de la papila con hemorragias radiadas y punteadas dentro del area papilar $y$ en el declive del mismo; mácula edematosa, con exudados en forma radiada; hasta la periferia la retina no adquiere caracteres normales; la circulación tiene el mismo aspecto que la del O.D. (Dr. Lloberas). El psiquismo de la enferma parece bastante normal, aunque se manifiesta muy deprimida y llora continuamente desde que ha empezado a notar que también se le "nubla" el ojo izquierdo. Urea en la sangre 0,73 $\mathrm{g}$ por mil. En la orina, ghucosa y albrimina negativas. Velocidad de sedimentación 21 y 13. Pruebas de eliminación normales. Radiografias del craneo normales. 
Ventriculografia: se puncionan ambos ventrículos laterales que son hallados en posición normal.

Intervención (Dr. Tolosa) - Craniectomia subtemporal derecha; abertura de la dura en estrella; sutura habitual de los planos aponeuróticos y tegumentarios. Curso posoperatorio - La enferma queja hipertermia y cefaleas debidas probablemente a una infección gripal. La herida operatoria presenta aspecto normal y la región craniectomizada abomba ligeramente. Es dada alta al cabo de 15 dias $\sin$ haberse modificado favorablemente el fondo de ojo. Se indica la conveniencia cie una nueva intervención (gangliectomia y esplancnicectomia lumbar) pero la enferma desaparece.

Puede considerarse este caso como un ejemplo típico de hipertensión esencial maligna en que el cuadro dominante correspondia a la hipertensión endocraneal por la encefalopatia que hizo pensar en la posibilidad de un tumor cerebral. A pesar de la importancia de las manifestaciones cerebrales, la participación renal era muy limitada.

En algunas de nuestras observaciones el cuadro de encefalopatia seudotumoral va precedido de algunos episodios angiospásticos cerebrales con ictus apopletiformes transitorios. En los casos que siguen, faltan manifestaciones cardiorrenales, coincidiendo las alteraciones de fondo de ojo con el cuadro de hipertensión maligna.

Caso 4 - Josefa S., de 56 años, sin antecedentes familiares patológicos importantes. Frecuentes cefalalgias. Hace un año sufrió intensos dolores abdominales, que fueron atribuidos a una afección hepática. Vómitos de tipo cerebral por las mañanas. Las cefalalgias se han acentuado, presentando crisis paroxísticas aproximadamente cada dos meses. También refiere que a menudo se le enturbia la vista. Hace un mes y inedio crisis intensa vertiginosa con vómitos, que le obligó a permanecer acostada durante 24 horas; perdió casi por completo el conocimiento; dolor intenso en la región occipital: A los 20 dias, nueva crisiz apopletiforme en que permaneció 4 dias sin conocimiento. Según refiere el médico de cabecera, hubo algunos signos de irritación meningea (rigidez de nuca y ligero Kernig), pero sin fiebre.

Examen clínico-neurológico - Fondo de ojo: en O.D., manchas corneales cicatrizales de úlceras antiguas; miopia fuerte con astigmatismo; coroiditis miópica con focos diseminados; retinitis hemorrágica con hemorragias retinianas en huso; estasis papilar moderado; campo visual reducido y con escotomas; la agudeza visual con corrección es de 0,1 . En O.I., mancha corneal mas reducida que en O.D.; miopia de 4 dioptrias; coroiditis miópica; extensas hemorragias retinianas; marcado estasis papilar y campo visual alterado; la agudeza visual con corrección es de 0,2 (Dr. Ariza). La exploración neurológica da resultado negativo. Presión arterial 24-14. Urea en la sangre $0,33 \mathrm{~g}$. En la orina, albúmina y glucosa negativas. Liquido cefalorraquideo: ligera hipertensión (40 en el Claude), pero el examen quimico y reacciones son normales.

Caso 5 - Valentin F., de 47 años, sin antecedentes patológicos familiares importantes. Es una hipertensa desde hace muchos años. Se ha quejado siempre de cefalalgias. Hallándose en la playa y después de haber tomado el sol durante más de una hora, crisis apoplética con pérdida de conocimiento, permaneciendo en coma completo durante más de cinco horas.

Examen clínico-neurológico - La paciente es reconocida por nosotros a los tres dias del accidente, siendo su estado actual el siguiente: enferma bien nutrida, mas bien obesa, en un estado constante de excitación, quejandose de faertes 
dolores de cabeza; hay momentos en que parece hallarse en un estado de psiquismo confusional. El examen ocular mostra borrosidad de los contornos de ambas papilas opticas; intenso nistagmo de amplias oscilaciones. Aunque es dificil de explorar, dada su agitación permanente, se aprecia marcada ataxia en las extremidades superiores $y$ inferiores. Reflejos rotulianos $y$ aquileos vivos. Signo de Babinski dudoso. Sensibilidad y resto de pares craneales normales. Presión arterial: 23-11. No albuminuria. Pruebas de eliminación renal normales. Líquido cefalorraquidio: hipertensión evidente (45 en el Claude acostada), pero con composición y reacciones normales. El curso es sumamente lento, of reciendo remisiones y agravaciones, llegando a encontrarse en estado semicomatoso varias veces durante algunas horas. Permanece durante más de un mes en este estado, recuperandose muy lentamente sobre todo la marcha, que es marcadamente titubeante todavia durante muchas semanas. Persiste todavia cierto estado de puerilismo y lentitud en asociación de ideas.

Es probable que en la provocación de la crisis influyera en este caso el factor insolación. Se creyó en la posibilidad de una hemorragia subaracnoidea, por lo que, a pesar de un comienzo de estasis papilar, se practicó una punción lumbar que solo nos señaló una hipertensión endocraneal moderada. Se trata pues de una enferma que desarrollo, despues de un periodo prolongado de cefalalgias, una sintomatologia poco precisa de fosa posterior o de tronco encefálico.

Queremos citar, finalmente, una observación en que, ante la intensidad de los vómitos, cefalalgias y estasis papilar, suponimos de momento nos encontrábamos ante un tumor cerebral.

Caso 6 - 'Teresa B., de 21 años, sin antecedentes personales ni familiares patológicos importantes desde el punto de vista neurológico. Desde hace un año intensas cefalalgias de localización predominante frontal, que han ido aumentando progresivamente en intensidad. Hace ocho días le pareció que le habia entrado un cuerpo extraño en el ojo, perdiendo bastante vista, pero volvió a recobrarla pasadas algunas horas. Presenta también, desde hace algunas semanas, náuseas y vómitos.

Examen clínico-neurológico - Faltan signos patológicos neurológicos si se exceptuan la abolición de reflejos abdominales y cierto grado de hiperreflexia rotuliana y aquilea. Pares craneales normales a excepción del segundo en que se observa edema papilar muy intenso y disminución considerable de la agudeza visual del ojo derecho; campo visual normal. Por la radiografia de cranbo se pone de manifiesto cierto grado de diastasis de las suturas. Punción lumbar: gran hipertensión del líquido cefalorraquideo del que se extrae una cantidad muy limitada a fin de poder examinar solamente las cifras de albúmina y células, que son normales asi como las reacciones de las globulinas. Aunque se sospecha un tumor cerebral es enviada al oftalmólogo, el cual nos indica que el caracter de las lesiones de fondo de ojo hace creer en la posibilidad de una retinitis albuminúrica de caracter grave. Interrogada de nuevo, la enferma recuerda que alguna vez se le habian hinchado ligeramente los tobillos. Antes de pasar al internista podemos comprobar la hipertensión arterial y una cifra moderada de albúmina en la orina. Segūn referencias, fallece a los ocho meses, de uremia.

\section{SUMARIO Y CONCLUSIONES}

Se describe con el nombre de encefalopatia hipertensiva seudotumoral aquellos casos en que la hipertensión arterial maligna origina un cuadro 
de hipertensión endocraneal que puede ir asociado a signos focales cere: brales simulando un proceso expansivo intracraneal.

Se considera a la encefalopatia hipertensiva seudotumoral como una complicación relativamente frecuente de la hipertensión maligna arterial primitf́va aunque también puede ser provocada a consecuencia de otros procesos causantes de hipertensión arterial (endocrinos, renales, vasculares, etc.).

Se describen algunas observaciones de encefalopatia hipertensiva en que se planteó el diagnostico diferencial con un tumor cerebral. Los signos clinicos focales (sindrome de Foster-Kennedy, sintomas cerebelosos, etc.) aumentaron en algunos casos las dificultades diagnósticas.

Se señala la importancia de los exámenes complementarios especialmente de la arteriografia cerebral y neumoencefalografia que han permitido casi siempre evitar errores diagnósticos.

\section{SUMMARY}

Some cases of malignant arterial hypertension are reported as "pseudotumoral hypertensive encephalopathy" with a clinical picture of elevated intracranial pressure, sometimes associated with localizing symptoms, simulating brain tumor.

Pseudotumoral hypertensive encephalopathy is relatively frequent in malignant arterial hypertension. It can also be provoked by other disturbances causing arterial hypertension (hormonal, renal, vascular, etc.).

Some cases of hypertensive encephalopathy are reported. The focal symptoms (Foster-Kennedy syndrome, signs of the posterior fossa, etc.) have been increasing difficulties.

The importance of cerebral arteriography and pneumoencephalography is stressed in most of cases avoiding erroneous diagnosis.

\section{BIBLIOGRAFIA}

1. Alsina Bofill - Comunicación personal.

2. Bordley, J. F. y Baker, B. M. - Arterioesclerosis of the cerebral vessels and pathogenesis of hypertension. Bull. Johns Hopkins Hosp., 39:229-264 (Octubre) 1936.

3. Cañizo, A. del - Estudio Clinico de la Hipertensión Arterial. Salvat Editores, Barcelona-Buenos Aires, 1942.

4. Cammarotta, A. y Matera, R. F. - Diagnóstico diferencial entre la encefalopatia hipertensiva arterial y el tumor cerebral. Dia Méd., 17:692-697 (9 Julio) 1945.

5. Davison, G. y Brill, N. -- Essential hypertension and chronic hypertensive encephalopathy. Ann. Int. Med., 12:1766-1781 (Mayo) 1939. 
6. Gispert, I. de - Afecciones seudotumorales cerebrales. Rev. Españ. de OtoNeuro-Oftalmol., 1:391-416 (Mayo) 1944.

7. Gispert, I. de - Sobre el sindrome de Cushing. Med. Esp., 41:7-14 (Julio) 1942.

8. Goodman, L. - Recurrent hypertensive cerebral thrombosis. Arch. Neurol. a. Psychiat., 62:445-478 (Octubre) 1949.

9. Griep, A. e col. $\rightarrow$ 'The prognosis in arterial hypertension. Am. J. M. Sc., 221:239-255 (Marzo) 1951.

10. Griffith, R. S. - Hypertensive encephalopathy simulating brain tumor. M. Clin. North America, 17:827-835 (Noviembre) 1933.

11. Holub, K. - Ungeklärte neurochirurgische Fälle. Wien. Med. Wochnschr., 3:349-352 (4 Junio) 1948.

12. Ketterer - Cit. por Goodman 8 .

13. Lopez Fernandez, J. - La hipertensión arterial forma de pseudo tumor cerebral. Rev. Méd. Cubana, 59:876-895 (Diciembre) 1948.

14. Mc Alpine, D. - Hypertensive cerebral attack. Brit. M. J., 2:990-993 (Noviembre) 1935.

15. Oppenheimer, B. S. y Fishberg, A. M. - Hypertensive encephalopathy. Ann. Int. Med., 41:264-278 (Febrero) 1928.

16. Pickering, L. - 'Transient cerebral paralisy in hypertension. J.A.M.A., 137: 423-430 (29 Mayo) 1948.

17. Puech, P. y Thierry, M. - Hypertension arterielle et hypertension intracranienne. Rev. Neurol., 65:289 (Ábril) 1936.

18. Scheinker, I. M. - Hypertensive encephalopathy. Monatsschr. f. Psychiat. u. Neurol., 102:159-190, 1940 .

19. 'Thiebault, F. - Anatomic-clinical study of cerebral form of arterial hypertension, renal, cardiac and cerebral lesions. Ann. de Med., 45:184-215 (Marzo) 1939.

Lauria 74 - Barcelona - España. 